Research Article

\title{
Assessment of resectability in carcinoma pancreas using multi-detector computed tomography with surgical correlation
}

\author{
Anuraj Appukuttan* \\ Department of General Surgery, Govt. Medical College, Thiruvananthapuram, Kerala, India \\ Received: 29 January 2016 \\ Revised: 07 March 2016 \\ Accepted: 17 March 2016 \\ *Correspondence: \\ Dr. Anuraj Appukuttan, \\ E-mail: arsa25@gmail.com \\ Copyright: (c) the author(s), publisher and licensee Medip Academy. This is an open-access article distributed under \\ the terms of the Creative Commons Attribution Non-Commercial License, which permits unrestricted non-commercial \\ use, distribution, and reproduction in any medium, provided the original work is properly cited.
}

\begin{abstract}
Background: Pancreatic cancers are considered a dreaded disease owing to the grave tumour biology and poor overall survival. The objective of this study was prospectively evaluating the ability of multi detector computed tomography (MDCT) to predict resectability of pancreatic cancer on the basis of surgical correlation.

Methods: 72 consecutive patients admitted to the departments of General Surgery and Surgical Gastroenterology with pancreatic cancer underwent preoperative contrast enhanced triple phase 128 slice MDCT- pancreatic protocol. Out of this 31 patients deemed resectable and underwent surgery. The operative resectability of tumour in terms of vascular invasion, local spread and abdominal metastasis was assessed. Surgical findings were reviewed and compared with pre-operative MDCT results.

Results: Of 31 tumours, considered to be resectable at MDCT evaluation, 25 were completely resected and six were found to be unresectable at surgery, yielding a positive predictive value of $80.6 \%$ with six false-negative results for overall resectability. None of 31 evaluated patients showed any evidence of vascular invasion, local spread or abdominal metastasis at MDCT examination. However out of the six inoperable patients, three had vascular invasion, three had evidence of local spread and one had small liver metastasis detected during surgery. Thus MDCT yielded a negative predictive value of $90.3 \%$ (28/31) for identifying the vascular invasion as well as for detection of local spread and a negative predictive value of $96.8 \%$ (30/31) at detecting abdominal metastases.

Conclusions: MDCT is an effective pre-operative tool for assessing resectability with a good positive predictive value for overall resectability in pancreatic adenocarcinoma.
\end{abstract}

Keywords: MDCT, Resectability, Pancreatic tumour

\section{INTRODUCTION}

Pancreatic cancers are considered a dreaded disease owing to the grave tumour biology and poor overall survival. Although pancreatic cancer accounts for only $2 \%$ of new cancers in the United States, it accounts for the 5 th leading cause of cancer deaths with approximately 30,300 new cases and 29,700 deaths reported in 2002. Because of its silent course, late clinical symptoms and rapid growth patterns, it has been named the "silent killer". ${ }^{1-3}$
About 15 to $20 \%$ of patients have resectable disease at the time of presentation. Surgical resection offers the only chance or cure with reported 5 year survival rates of $8 \%$ to $21 \%$. The main role of preoperative staging procedures is to distinguish between potentially resectable and unresectable patients so that unnecessary surgical procedures may be avoided; survival benefits can be achieved only in patients in whom the tumour can be completely resected. Contrast enhanced, helical computed tomography (HCT) and more recently multidetector computed tomography (MDCT) have been widely accepted as the imaging technique of choice for the staging of pancreatic adenocarcinoma. The 
introduction of helical CT improved our ability to appropriately stage disease; however, a significant number of patients are still incorrectly diagnosed as having resectable tumour on CT only to be unresectable at surgery. The proportion of patients undergoing unnecessary laparotomy may vary between $21 \%$ and $44 \% .^{4-8}$

MDCT-pancreatic protocol study remains the favoured investigation for pre-operative staging of the disease. MDCT has a sensitivity of $82 \%$ and specificity of $75 \%$ in pancreatic cancers. The accurate determination of resectability in patients with pancreatic cancer is the most important contribution of pre-operative staging, the goal being to reduce needless surgery to a minimum. Laparotomy in patients with pancreatic cancer carries significant peri-operative morbidity of $20 \%-30 \%$ and mortality of up to $5 \%$ for Whipple's operation even in expert hands. 9,10

Moreover prognosis is not improved for patients whose tumours are resected with positive margins or vascular invasion. On the other hand for most patients with unresectable lesions, laparotomy for palliative procedures has become unnecessary because of recent advances in endoscopic and percutaneous methods of biliary and duodenal drainage.

The introduction of MDCT has offered a substantial improvement in volume coverage speed with no loss of image quality facilitating optimal pancreatic parenchymal and per pancreatic vascular enhancement. In addition, MDCT facilitates the generation of multiplanar reconstructions, such as curved planar reformations, providing the potential to improve the detection and staging of pancreatic adenocarcinoma. ${ }^{11-15}$

One in five patients deemed resectable by MDCT are found to be unresectable on surgical exploration; 20\%$40 \%$ of patients in whom MDCT findings suggest resectability are in fact not. Having impressed upon the importance of accurate pre-operative CT staging and the difference in the outcome on complete resection, it is clear that CT is never $100 \%$ predictable. $^{4}$

The purpose of this study is to evaluate, prospectively, the ability of MDCT to predict resectability of pancreatic cancer on the basis of surgical outcome. As a correct preoperative staging of resectability in carcinoma pancreas is the core in defining the treatment strategy especially curative resection, it is imperative to critically analyze the predictive power of MDCT.

\section{METHODS}

This was a prospective observational study, conducted in the departments of General Surgery and Surgical Gastroenterology in our tertiary care hospital over a period of 8 months. Ethical clearance was obtained from the Institutional Human Ethics Committee prior to the study. Confidentiality and anonymity of the patient's information was maintained during and after the study.

\section{Patients}

72 patients were admitted to the Surgical Gastroenterology and General Surgery departments during the study period. All the patients had undergone triple phase MDCT 128 slice pancreatic protocol either prior to or after admission. 31 patients deemed resectable for carcinoma pancreas on MDCT reports, meeting all the inclusion criteria were included in the study. Criteria defining resectability status (Radiological and Surgical) includes Tumors with (a) No distant metastases evaluated by the standard clinical and radiological methods in the pre-operative work up (b) Clear fat planes/ no involvement of celiac axis, hepatic artery, superior mesenteric artery (c) No involvement of superior mesenteric vein and/or portal vein or venous involvement of superior mesenteric vein, portal vein, with abutment, encasement, or obstruction by tumor thrombus but with suitable proximal and distal vessel length to allow for safe resection and reconstruction. ${ }^{16}$

\section{CT technique and image analysis}

All examinations were performed on 128 slice Siemens MDCT. All patients drank $500 \mathrm{ml}$ of water to demarcate the duodenum and delineate the pancreatic head. $50 \mathrm{ml}$ of iodinated intra venous contrast - Iohexol $(350 \mathrm{mg} / \mathrm{ml})$ was injected and triple phase MDCT images were taken at 20, 60 and 120 seconds for arterial, portal and venous phases respectively. Majority of the patients had a delayed imaging at 180 seconds to determine the excretion of contrast. Each pancreatic cut section was taken at $0.6 \mathrm{~mm}$.

Axial images as well as curved and multi planar reconstructions were reviewed. Radiological criteria of resectability included: the absence of liver metastases and peritoneal carcinomatosis, tumour infiltration in contact with less than $180^{\circ}$ of the circumference of the walls of major arteries (celiac trunk, hepatic artery, superior mesenteric artery) and involvement of less than $180^{\circ}$ of the circumference of the portal vein or the superior mesenteric vein. The MDCT findings were correlated with surgical outcomes to assess accuracy in predicting resectability.

A written informed consent was obtained from the patient and the caretaker. Patient's clinical and investigatory reports were recorded. All the patients in the study group were followed up during surgery which took place no more than 15 days from the date of CT scanning. The operative resectability of tumour in terms of metastasis, local spread and vascular invasion was assessed during laparotomy. The specimens sent for histopathology were followed up for confirmation of malignancy. Finally the surgical findings were compared with the pre-operative CT reports and the predictive power of MDCT was assessed with surgical outcome as reference standard. 
The clinical details in terms of demographic properties, CA 19-9 values and liver function tests were evaluated and their relation with intra-operative resectability was also studied.

\section{RESULTS}

All the 72 patients admitted to the Surgical Gastroenterology and General Surgery departments with carcinoma pancreas had underwent triple phase MDCT 128 slice pancreatic protocol either prior to or after admission. After radiological and clinical assessment, 41 $(56.9 \%)$ patients were not suitable for curative surgery, while $31(43.1 \%)$ patients had favourable radiological findings with good performance status and were eligible for surgical resection. These 31 patients were evaluated and data were analyzed.

Table 1: Pre-operative staging of MDCT in comparison with surgical outcome.

\begin{tabular}{|c|c|c|c|c|c|}
\hline & \multicolumn{2}{|c|}{ MDCT } & \multicolumn{2}{|c|}{ Surgery } & \multirow[b]{2}{*}{$\begin{array}{l}\text { NPV } \\
(\%)\end{array}$} \\
\hline & (-ve) & $(+v e)$ & $(-v e)$ & $(+v e)$ & \\
\hline $\begin{array}{l}\text { Vascular } \\
\text { invasion }\end{array}$ & 31 & - & 28 & 3 & 90.3 \\
\hline $\begin{array}{l}\text { Local } \\
\text { spread }\end{array}$ & 31 & - & 28 & 3 & 90.3 \\
\hline $\begin{array}{l}\text { Liver } \\
\text { metastasis }\end{array}$ & 31 & - & 30 & 1 & 96.8 \\
\hline
\end{tabular}

Of 31 tumours, considered to be resectable at MDCT evaluation, 25 were completely resected (20 patients underwent pancreaticoduodenectomy, 5 underwent distal pancreatectomy). Six tumours were found to be unresectable at surgery.

The reasons for unresectability were vascular involvement, local spread and distant metastasis - three patients had vascular involvement, two patients had local spread and one patient had local spread as well as metastasis. The positive predictive value for pancreatic carcinoma resectability was $80.6 \%$ with six falsenegative results. The standard error for positive predictive value was 7.102 with confidence interval ranging from 66.4 to 94.8 .

A total of three arteries and two veins were evaluated for vascular involvement: celiac trunk, superior mesenteric artery, common hepatic artery, portal vein and superior mesenteric vein.

None of 31 evaluated patients showed an evidence of vascular invasion at MDCT examination. However out of the six inoperable patients, three had vascular invasiontwo cases with portal vein encasement and one with superior mesenteric artery invasion. Thus MDCT yielded a negative predictive value of $90.3 \%$ (28/31) for identifying the vascular invasion.

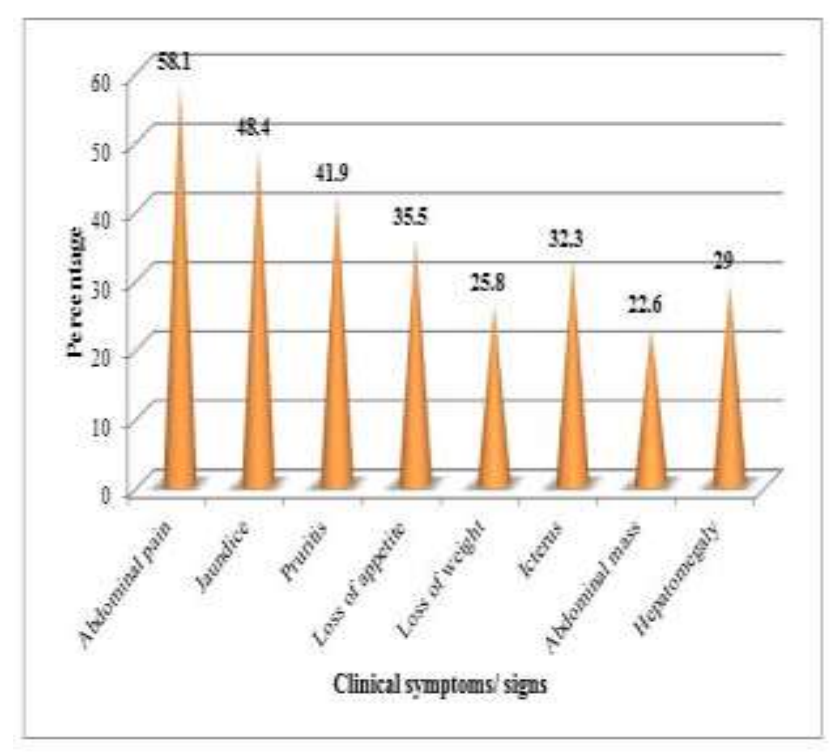

Figure 1: Clinical presentation of the patients in study population.

Local spread on to the retro peritoneum with fixity, lesser sac involvement and duodenal infiltration were evaluated. Of the total 31 patients none had any evidence of local spread at MDCT examination. However three of the six unresectable patients had evidence of local spread, detected during surgery. Two cases had fixity of mass on to the retro peritoneum, while one had duodenal infiltration. For detection of local spread, MDCT yielded a negative predictive value of $90.3 \%$ (28/31).

Metastases to the liver, peritoneum deposits and ascites were evaluated. None of the 31 patients had any evidence of metastases on MDCT. One tumour was found to be unresectable at surgery because of small liver metastases. The lesion size averaged $5 \mathrm{~mm}$. They did not show typical attenuation and were not disclosed even after careful review of CT images. Thus MDCT yielded a negative predictive value of $96.8 \%$ (30/31) at detecting abdominal metastases.

The age range of study population was 40 to 76 years. Mean age of the patients was 54.97 years. Number of patients were maximum $(35.5 \%)$ in the age interval of 40 49 years. Majority of patients were females $(54.8 \%)$. Out of 31 patients evaluated, $11(35.5 \%)$ patients had diabetes mellitus, $8(25.8 \%)$ patients had hypertension and 4 (12.9\%) patients had dyslipidemia.

The various presenting complaints of patients were jaundice, pruritus, abdominal pain, loss of appetite, loss of weight etc. Among these the most common was abdominal pain $(58.1 \%)$ followed by jaundice $(48.4 \%)$ and pruritus $(41.9 \%)$. On examination, $32.3 \%$ of patients had icterus, $22.6 \%$ of patients had mass per abdomen on presentation and $29 \%$ of patients had hepatomegaly. (Total exceeds $100 \%$, since the patients were having more than one symptom/ sign). CA 19-9 levels was 
elevated in majority of patients. It was $>100 \mathrm{U} / \mathrm{ml}$ in 14 $(45.2 \%)$ patients and was between $40-100 \mathrm{U} / \mathrm{ml}$ in 9 (29\%) patients. CA 19-9 was not elevated in $8(25.8 \%)$ patients. Of the six inoperable patients at surgery, four (66.7\%) patients had CA 19-9 levels >300U/ml. Serum bilirubin was elevated in $15(48.4 \%)$ patients and serum ALP was elevated in $18(58.1 \%)$ patients. $19.4 \%$ of patients had elevated SGPT and SGOT. $16.1 \%$ of patients had anaemia at the time of presentation.

The tumour was located in the pancreatic head and neck region in 25 patients $(83.87 \%)$ and in the body and tail of the pancreas in 6 patients $(16.12 \%)$. The mean tumour size was $3.6 \mathrm{~cm}$ (range 1.5-6.5 $\mathrm{cm}$ ) on the basis of pathologic findings for the 25 patients who underwent resection and on the basis of CT measurement in the 6 nonsurgical patients.

\section{DISCUSSION}

During Pancreatic cancer is a dreaded disease, majority of the patients presenting at a late stage of the tumour precluding them from any curative therapy. Surgery is the only curative modality and this depends on the preoperative MDCT assessing its resectability. However one in five patients deemed resectable by CT is in fact inoperable. Hence it is crucial to critically analyze the predictive power of MDCT in our institution. This study was undertaken to assess the ability of MDCT to predict the resectability of pancreatic cancer.

In the present study, majority of the patients belong to the age group of $40-49$ years $(35.5 \%)$ and the mean age of the patient in this study was 54.97 years. Mean age of the patient was almost similar with the study by Aziz AM et al. In the study by Olivie D et al and Manak E et al mean age of the patient was 63 and 64.7 years respectively. Female predominance $(54.8 \%)$ was observed in the present study. But male predominance was observed in the studies by Aziz AM et al (59.4\%) as well as by Manak et al $(54.2 \%)$. But the study by Olivie D et al, observed equal distribution of males and females. ${ }^{17-19}$

Most of the patients were presented with abdominal pain $(58.1 \%)$ followed by jaundice $(48.4 \%)$. CA 19-9 levels were elevated in majority $(74.2 \%)$ of the patients. Among the inoperable patients $66.7 \%$ (4/6) had CA 19-9 levels of more than $300 \mathrm{U} / \mathrm{ml}$.

In the present study majority $(83.87 \%)$ of the tumours were located in the pancreatic head and neck followed by body and tail of the pancreas (16.12\%). This finding was consistent with the studies by Manak E et al, and Vargas et al, as they observed that $90 \%$ of pancreatic tumours were located in the pancreatic head region. The mean tumour size was $3.6 \mathrm{~cm}$ (range, $1.5-6.5 \mathrm{~cm}$ ) in this study. The mean tumour size was reported as $2.2 \mathrm{~cm}, 2.8 \mathrm{~cm}$, $4.4 \mathrm{~cm}$ in various studies. ${ }^{17-20}$
In a group of 31 patients, the present study observed a positive predictive value of $80.6 \%$ for resectability in pancreatic carcinoma. Of the 31 tumours considered resectable at MDCT evaluation, 25 were completely resected. Six tumours were found to be unresectable at surgery. This was almost similar with the observations of few other studies. The PPV was high in the present study compared with the studies by Ellsmere et al (61\%) and Smith et al $(56 \%){ }^{21-24}$

According to the studies by Aziz AM et al and Olivie D et al, when they compared MDCT results to surgical outcome, the positive predictive value of MDCT for surgically resectable disease was $100 \%$. In a study by Manak E et al, the PPV for pancreatic adenocarcinoma resectability was $91 \%$ with four false-negative results. Predictive values of HCT for resectability ranging from $56 \%$ to $79 \%$ have been reported. This shows the improvement in prediction of resectability by MDCT. . $^{-}$ $8,17-19,25,26$

The common reason for unresectability was vascular invasion, local spread and metastases. In the present study MDCT yielded a NPV of $90.3 \%$ for detecting vascular invasion and local spread, and NPV of $96.8 \%$ for detecting abdominal metastases. Soriano et al, examined the efficacy of CT, endoscopic ultrasound, magnetic resonance imaging and angiography and they concluded that CT had the highest accuracy for assessing the extent of the local tumour (73\%), local extension $(73 \%)$, vascular invasion $(83 \%)$, metastases $(88 \%)$ and resectability $(83 \%)$. The reported accuracy for determining vascular invasion using single-detector helical CT is in the range of 70-95\%.,27-30

In a study by Vargas et al, they found that for detecting vascular invasion by tumour on a vessel-by-vessel basis, MDCT yielded a negative predictive value of $100 \%$ (108/108) with no false-negative findings. A study by Aziz AM et al, the comparison between the MDCT and surgical result, showed that the NPV for vascular invasion was $86.6 \%$ and for liver metastases $96.7 \%$. Manak E et al reported that MDCT yielded a NPV of $98 \%$ for vascular invasion and $96 \%$ for liver metastases. ${ }^{17,19,20}$

This study does present some limitations. The number of patients included in the study group was small and it is possible that the results would differ with a larger series. One bias is that we limited our study group to those who had their CT study performed at our institution.

Finally, our study focused on patients who had undergone surgery, so a selection bias toward potentially resectable tumours is introduced because only patients with such tumours tend to be referred for surgical evaluation. 


\section{CONCLUSION}

MDCT is an effective pre-operative tool for assessing resectability with a good positive predictive value for overall resectability in pancreatic adenocarcinoma.

\section{ACKNOWLEDGEMENTS}

I extend my sincere gratitude to my colleagues in the department of general surgery and teaching staff of the department of surgical gastroenterology for their help and valuable suggestions. I am extremely grateful to the patients who agreed to be part of this study, without whose co-operation this study would have been impossible.

Funding: No funding sources

Conflict of interest: None declared

Ethical approval: The study was approved by the institutional ethics committee

\section{REFERENCES}

1. Jemal A, Tiwari RC, Murray T, Ghafoor A, Samuels A, Ward E, et al. Cancer Statistics 2004. CA Cancer J Clin. 2004;54(1):8-29.

2. Kloppel G, Solcia E, Longnecker DS, Capella C, Sorbin LH. Histological typing of tumours of the exocrine pancreas. In: world health organisation. International histological classification of tumours. $2^{\text {nd }}$ ed. Berlin Heidelberg, New York: Springer; 1996:1-7.

3. Pour PM. The silent killer. Int $\mathrm{J}$ Pancreatol. 1991;10:103-4.

4. Li D, Xie K, Wolff R, Abbruzzese JL. Pancreatic cancer. Lancet. 2004;363:1049-57.

5. Neoptolemos JP, Stocken DD, Friess H, Bassi C, Dunn JA, Hickey H, et al. A randomized trial of chemoradiotherapy and chemotherapy after resection of pancreatic cancer. $\mathrm{N}$ Engl $\mathrm{J}$ Med. 2004;350:1200-10.

6. Valls C, Andía E, Sanchez A, Fabregat J, Pozuelo O, Quintero JC, et al. Dual-phase helical CT of pancreatic adenocarcinoma: assessment of resectability before surgery. Am J Roentgenol. 2002;178:821-6.

7. Bluemke DA, Cameron JL, Hruban RH, Pitt HA, Siegelman SS, Soyer P, et al. Potentially resectable pancreatic adenocarcinoma: spiral CT assessment with surgical and pathologic correlation. Radiology. 1995; 197:381-5.

8. Diehl SJ, Lehmann KJ, Sadick M, Lachmann R, Georgi M. Pancreatic cancer: value of dual-phase helical CT in assessing respectability Radiology. 1998;206:373-8.

9. Schulick RD, Cameron JL. Cancer of pancreas and other periampullary cancers. In: Zinner MJ, Ashley SW, eds. Maingot's Abdominal operations. $11^{\text {th }}$ ed, New York: McGraw Hill. 2007:1038-1039.
10. Warshaw AL, Castillo FC. Pancreatic cancer. N Engl J Med. 1992;326(7):455-65.

11. Hu H, He D, Foley D, Fox SH. Four multidetectorrow helical CT: image quality and volume coverage speed. Radiology. 2000;215:55-62.

12. McNulty NJ, Francis IR, Platt JF, Cohan RH, Korobkin M, Gebremariam A. Multidetector-row helical CT of the pancreas: effect of contrastenhanced multiphasic imaging on enhancement of the pancreas, peripancreatic vasculature, and pancreatic adenocarcinoma. Radiology. 2001;220:97-102.

13. Murcia NM, Jeffrey RB, Beaulieu CF, Li KCP, Rubin GD. Multidetector CT of the pancreas and bile duct system: value of curved planar reformations. Am J Roentgenol. 2001;176:689-93.

14. Prokesch RW, Chow LC, Beaulieu CF, Bammer R, Jeffrey RB. Local staging of pancreatic carcinoma with multidetector - row CT: use of curved planar reformations - initial experience. Radiology. 2002;224(3):764-8.

15. Fishman EK, Horton KM, Urban BA. Multidetector CT angiography in the evaluation of pancreatic carcinoma: preliminary observations. J Comput Assist Tomogr. 2000;24:849-53.

16. NCCN clinical practice guidelines in oncology V.2.2010. Available at http://www.nccn.org. Accessed on September 2010.

17. Aziz AM, Said T, Poovathumkadavil A, Almulla A. Using multidetector CT in predicting resectability of pancreatic head tumors: surgical and pathologic correlation. Journal of the Egyptian Nat Cancer Inst. 2010;22(4):233-9.

18. Olivié D, Lepanto L, Billiard JS, Audet P, Lavallée JM. Predicting resectability of pancreatic head cancer with multi-detector CT. Surgical and Pathologic Correlation. J Pancreas. 2007;8(6):753-8.

19. Manak E, Merkel S, Klein P, Papadopoulos T, Bautz WA, Baum U. Resectability of pancreatic adenocarcinoma: assessment using multidetectorrow computed tomography with multiplanar reformations. Abdom Imaging. 2009;34:75-80.

20. Vargas R, Murcia NM, Trueblood W, Jeffrey RB. MDCT in pancreatic adenocarcinoma: Prediction of vascular invasion and resectability using a multiphasic technique with curved planar reformations. Am J Roentgenol. 2004;182:419-25.

21. Laghi A, Iannaccone R, Catalano C, Carbone I, Sansoni I, Mangiapane F, et al. Multislice spiral computed tomography in diagnosis and staging of pancreatic carcinoma: Preliminary experience. Dig Liver Dis. 2002;34(10):732-8.

22. Maire F, Sauvanet A, Trivin F, Hammel P, O'Tool D, Palazzo L, et al. Staging of pancreatic head adenocarcinoma with spiral CT and endoscopic ultrasonography: An indirect evaluation of the usefulness of laparoscopy. Pancreatology. 2004;4(5):436-40.

23. Ellsmere J, Mortele K, Sahani D, Maher M, Cantisani V, Wells W, et al. Does multidetector-row 
CT eliminate the role of diagnostic laparoscopy in assessing the resectability of pancreatic head adenocarcinoma? Surg Endosc. 2005;19(3):369-73.

24. Smith SL, Basu A, Rae DM, Sinclair M. Preoperative staging accuracy of multidetector computed tomography in pancreatic head adenocarcinoma. Pancreas. 2007;34:180-4.

25. Procacci C, Biasiutti C, Carbognin G, Bicego E, Graziani R, Franzoso F, et al. Spiral computed tomography assessment of resectability ofpancreatic ductal adenocarcinoma: analysis of results. Digest Liver Dis. 2002;34:739-47.

26. Phoa SS, Tilleman EH, Delden OM, Bossuyt PM, Gouma DJ, Lameris JS. Value of CT criteria in predicting survival in patients with potentially resectable pancreatic head carcinoma. J Surg Oncol. 2005;91:33-40.

27. Soriano A, Castells A, Ayuso C, Ayuso JR, Caralt MT, Ginès MA, et al. Preoperative staging and tumor resectability assessment of pancreatic cancer: prospective study comparing endoscopic ultrasonography, helical computed tomography, magnetic resonance imaging, and angiography. Am J Gastroenterol. 2004;99(3):492-501.

28. Lu DS, Reber HA, Krasny RM, Kadell BM, Sayre J. Local staging of pancreatic cancer: criteria for unresectability of major vessels as revealed by pancreatic-phase, thin-section helical CT. Am J Roentgenol. 1997;168:1439-43.

29. O'Malley ME, Boland GW, Wood BJ, Castillo FDC, Warshaw AL, Mueller PR, et al. Adenocarcinoma of the head of the pancreas: determination of surgical unresectability with thinsection pancreatic-phase helical CT. Am J Roentgenol. 1999;173:1513-8.

30. Raptopoulos V, Steer ML, Sheiman RG, Vrachliotis TG, Gougoutas CA, Movson JS. The use of helical $\mathrm{CT}$ and CT angiography to predict vascular involvement from pancreatic cancer: Correlation with findings at surgery. AJR Am J Roentgenol. 1997;168:971-7.

Cite this article as: Anuraj A. Assessment of resectability in carcinoma pancreas using multidetector computed tomography with surgical correlation. Int Surg J 2016;3:701-6. 